\title{
Identificación de principales causas de contagio del Covid-19
}

\author{
DOI: https://doi.org/10.17981/bilo.3.1.2021.03 \\ Fecha de Recepción: 01/04/2021. Fecha de Publicación: 05/05/2021
}

\begin{abstract}
Altamar-Chacón, K.
kaltamar@cuc.edu.co
\end{abstract}

Monsalve-Villarreal, $M$.

mmonsalv7@cuc.edu.co

Vásquez-Hoyos, D.

dvasquez@cuc.edu.co

Alexander Troncoso-Palacio

atroncos1@cuc.edu.co

Departamento de Productividad e Innovación

Universidad de la Costa. Barranquilla, (Colombia)

\begin{abstract}
Resumen. El presente proyecto tiene como objetivo mostrar la situación de Barranquilla en tiempos de pandemia, en el cual se identificaron los motivos principales del contagio progresivo en el departamento. Se realizo mediante búsquedas en Google académico y en la biblioteca de la universidad como Scopus, con la información obtenida se implementaron herramientas de la calidad como, el Checklist, el histograma y el diagrama de Ishikawa, con los cuales se logró analizar las muertes desde el tiempo en que inicio a propagarse este virus en Barranquilla, además de las causas principales de su transmisión y cuáles fueron las prevenciones delegadas por el presidente de Colombia.
\end{abstract}

Palabras Clave: Covid 19, Causa-Efecto, Pandemia, Histograma

\section{Introducción}

El ministerio de salud es una entidad que tiene como función coordinar con las áreas y las entidades adscritas y vinculadas a la gestión de asuntos internacionales y de cooperación del sector de la salud. Teniendo en cuenta esta entidad, podemos definir el COVID-19 como un virus que surge de manera periódica en distintas partes del mundo, que afecta principalmente el sistema respiratorio, además de que le puede dar a cualquier persona, sean bebés, niños, jóvenes, adultos y adultos de la tercera edad. De acuerdo con la entidad, el Atlántico fue uno de los departamentos que avanzó rápidamente con índices de afectados por COVID-19, en el mes de Julio, ya teníamos 52.313 casos activos. Ya en el mes de agosto, el índice de activos fue de unos 59.101 casos. Y Actualmente los casos en el mes de octubre fueron de 68.621. La ciudad de Barranquilla es uno de los lugares de los cuales no cuenta con una preparación para este nivel de afectaciones, puesto que se está viendo afectado e involucrado la salud mental de aquellas personas profesionales de la misma área, debido a su alto índice y poca prevención al comienzo del contagio de este virus. La organización mundial de la salud fue una de las entidades que 


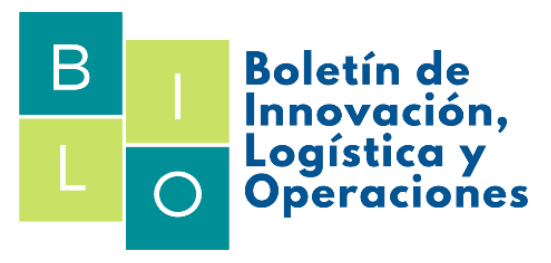

BILO Vol. 3 no. 1, Enero - Junio de 2021

hablo sobre el mismo tema dando recomendaciones necesarias para prevenir y evitar la propagación de esta enfermedad, con el objetivo de lograr la disminución del registro de afectados.

No conformes con todos estos casos, actualmente se maneja la desinformación para muchas personas, el mal manejo de los medios hace que las personas obtengan un mal manejo de la enfermedad, no sabiendo que esto es uno de los principales factores para el mejoramiento tanto emocional como el de la salud.

\section{Breve revisión de la literatura}

Algunas investigaciones como [1], [2] hacen, referencia al COVID-19 por su característica como lo es un cuadro de infección respiratoria. Dentro de la población de alto riesgo se encuentran adultos mayores, pacientes con comorbilidades cardiovasculares, alteraciones metabólicas, enfermedad pulmonar crónica, estados de inmunosupresión y profesionales de la salud también se presentan [3]. [4] Una serie de síntomas neurológicos leves inespecíficos que son notables en pacientes hospitalizados con COVID19, incluyendo dolor de cabeza , mareos, mialgia y / o fatiga, anorexia, anosmia y ageusia, aunque la epidemiología puede ser diferente en presentaciones ambulatorias más leves . Según [5], Hasta lo que va del milenio, se puede afirmar que esta es la pandemia más grande y larga que se ha vivido. Ninguna otra enfermedad infecciosa o epidemia, había logrado paralizar social y económicamente al mundo, detener los viajes internacionales, el turismo, eventos deportivos mayores entre otros múltiples impactos a los cuales ha conllevado el cierre de países y las cuarentenas establecidas con el fin de reducir la transmisión y reducir los decesos. Modelos propuestos, [6], [7], para auxiliar la toma de decisiones de los organismos gubernamentales de salud que deben determinar acciones para mitigar los efectos de la pandemia en la población. Se usaron los datos presentados por el Instituto Nacional de Salud el 26 de mayo de 2020 para Barranquilla y el departamento del Atlántico.

Por otro lado, [8], [9], [10] los registros oficiales, en este caso relacionados con las violaciones a los lineamientos nacionales sobre el comportamiento a seguir por la ciudadanía para evitar o reducir el contagio por COVID-19, permiten una sistematización uniforme del registro a nivel nacional, con cubrimiento de todas las regiones del país. En segundo lugar, permiten reunir un acumulado de registros que pueden analizarse desde una perspectiva censal y, en tercero, las posibles diferencias en la actuación por la región de las diferentes unidades de la Policía encargadas de detectar los incumplimientos que pueden constituirse en una variable en sí misma que se incluya en el análisis y la interpretación de los datos. [11], [12], [13] El coronavirus ha tenido un gran impacto en el mundo puesto ha colocado a las personas a vivir desafíos éticos, sociales y económicos y de forma externa en el medio ambiente. La pandemia ha traído consigo problemas de cuales todas las personas son afectada de una u otra manera, como psicológicamente, especialmente a aquellas personas que han estado todo el tiempo a la defensiva de este virus, creyendo en que simplemente es una exageración del gobierno. [14], [15], [16] indaga en el contexto actual de combate de la pandemia en los tiempos sociales que evidencian aún más las inequidades y desigualdades, demuestran la lucha de sobrevivencia de las clases y las reconfirmaciones de poderes, materializados en riesgos diferenciales al COVID-19, como el acceso a tratamiento. [17], [18]Tenemos la oportunidad de repensar y rearticular nuestras sociedades con la pandemia actual. Al mismo tiempo debemos replantear nuestras sociedades ya que la patología de la pandemia del COVID19 es resultado del desequilibrio ambiental, de la pérdida de biodiversidad y de la (re)emergencia de patógenos. [19, 20],La prevalencia de este virus sin duda, es algo impredecible, va más que todo en el comportamiento y actitud que tomen las personas a la hora de obedecer las prevenciones dadas por el ministerio de salud.

\section{Metodología}

Este proyecto se basa en estudios acerca de los casos activos del COVID-19 en la ciudad de Barranquilla durante la pandemia, en el que se empleó bases de datos confiables para que la información tenga alto rigor. El proceso de búsqueda se realizó de manera investigativa, sobre todos los casos ocurridos en

(C) The author; licensee Universidad de la Costa - CUC. 
Barranquilla, tomando referencias en artículos ya realizados en las plataformas Scorpus y Google académico e información expuesta por el DANE y el Ministerio de Salud Nacional. Para ello se implementaron palabras claves como: "Casos activos por Covid-19" y "Casos activos en Barranquilla". Teniendo en cuenta la relevancia actual de la información a examinar, está investigación fue limitada en estos 6 últimos meses, es decir, se tuvieron en cuenta los últimos documentos publicados del actual año.

También se basó en las investigaciones realizadas acerca de los casos por cada mes. Mediante esto se realizó un Checklist, en el cual se obtuvieron 8 indicadores, con el cual se realizó el Histograma para representar gráficamente las estadísticas de los afectados por el virus.

Mediante investigaciones realizadas, nos podemos dar cuenta que el COVID-19, que es un virus que se produjo en el año 2019-2, se transmite al entrar en contacto con otra persona afectada por medio de estornudos, tos o espira. Para analizar la propagación del virus, se realizó un diagrama de Ishikawa para observar las causas-raíces del presente problema.

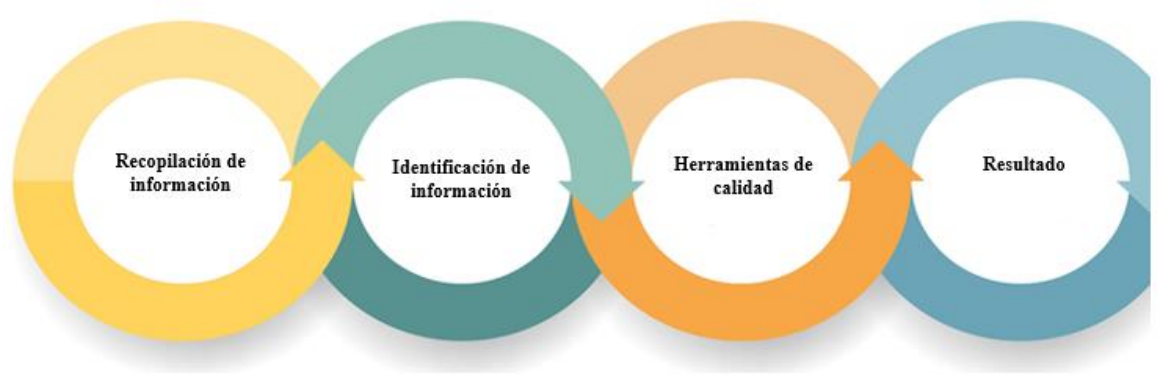

Figura 1: Diagrama adaptado del proceso de la metodología

\section{Análisis y resultados}

El COVID-19, es un virus que muta y se contagia rápidamente por todo el mundo, esta enfermedad hace parte de los virus SARS-coV y MERS-coV. Se originó en la ciudad de Wahuan en la provincia de Hubei, por aquellas personas que se relacionaban o tenían contacto en el mercado de Huana, en el cual ahí venden pescados, mariscos y animales vivos. Debido a esto pensaron que era un tipo de enfermedad como la neumonía, pero al cabo de un corto tiempo se dieron cuenta de que el número de personas infectadas y enfermas por el mismo caso aumentaba rápidamente. El primer caso reportado de muerte fue el 11 de enero. De ahí fue creciendo este virus, por personas que habían viajado al lugar y fueron transmitiéndolo a otras personas, puesto que su contagio es de contacto directo con la persona afectada. Teniendo en cuenta las investigaciones sobre el COVID-19, pudimos notar que tuvo un gran crecimiento en Barranquilla, a comienzos del mes de julio, debido a que las personas no acatan las normas o reglas establecidas por el gobierno. Para esto realizamos un Checklist sobre los meses y las muertes ocurridas desde el aislamiento establecido por el presidente de Colombia.

\begin{tabular}{|c|c|}
\hline \multicolumn{2}{|c|}{ COVID-19 } \\
\hline MESES & CASOS ACTIVOS \\
\hline Marzo & 1 \\
\hline Abril & 9 \\
\hline Mayo & 88 \\
\hline Junio & 681 \\
\hline Julio & 1,163 \\
\hline Agosto & 1,347 \\
\hline Septiembre & 1,396 \\
\hline Octubre & 1,398 \\
\hline
\end{tabular}

Figura 2. Checklist tomado del ministerio de salud

(C) The author; licensee Universidad de la Costa - CUC. 
Mediante los resultados obtenidos podemos notar los meses en los cuales entramos en aislamiento y los casos activos exactos en la ciudad de Barranquilla, con respecto a esto, se realizó un histograma para representarlo gráficamente y ver su incremento en cada mes de los casos activos por este virus.

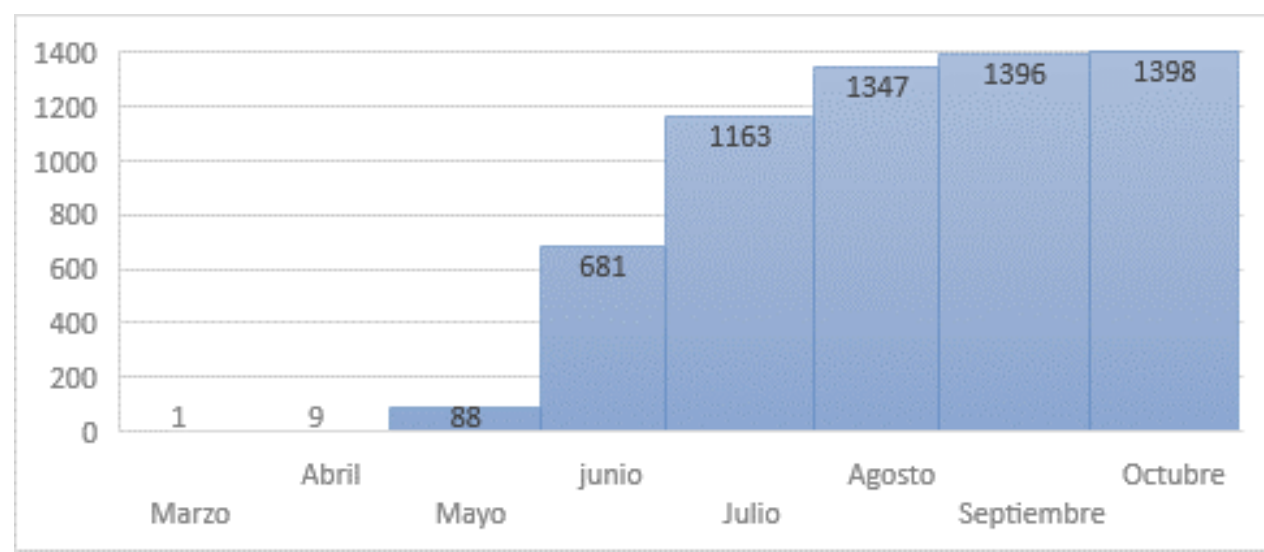

Figura 3. Histograma del incremento de casos activos tomado del ministerio de salud

Debido a la representación anterior podemos afirmar lo dicho en la introducción de este trabajo, sobre el gran número de incremento de ocurrencias en el mes de julio y agosto, ya para el mes de septiembre y octubre el índice de este virus bajo en un nivel considerable.

Por consiguiente, quisimos analizar la causa del crecimiento elevado del contagio de este virus, en el cual realizamos un Diagrama de Ishikawa.

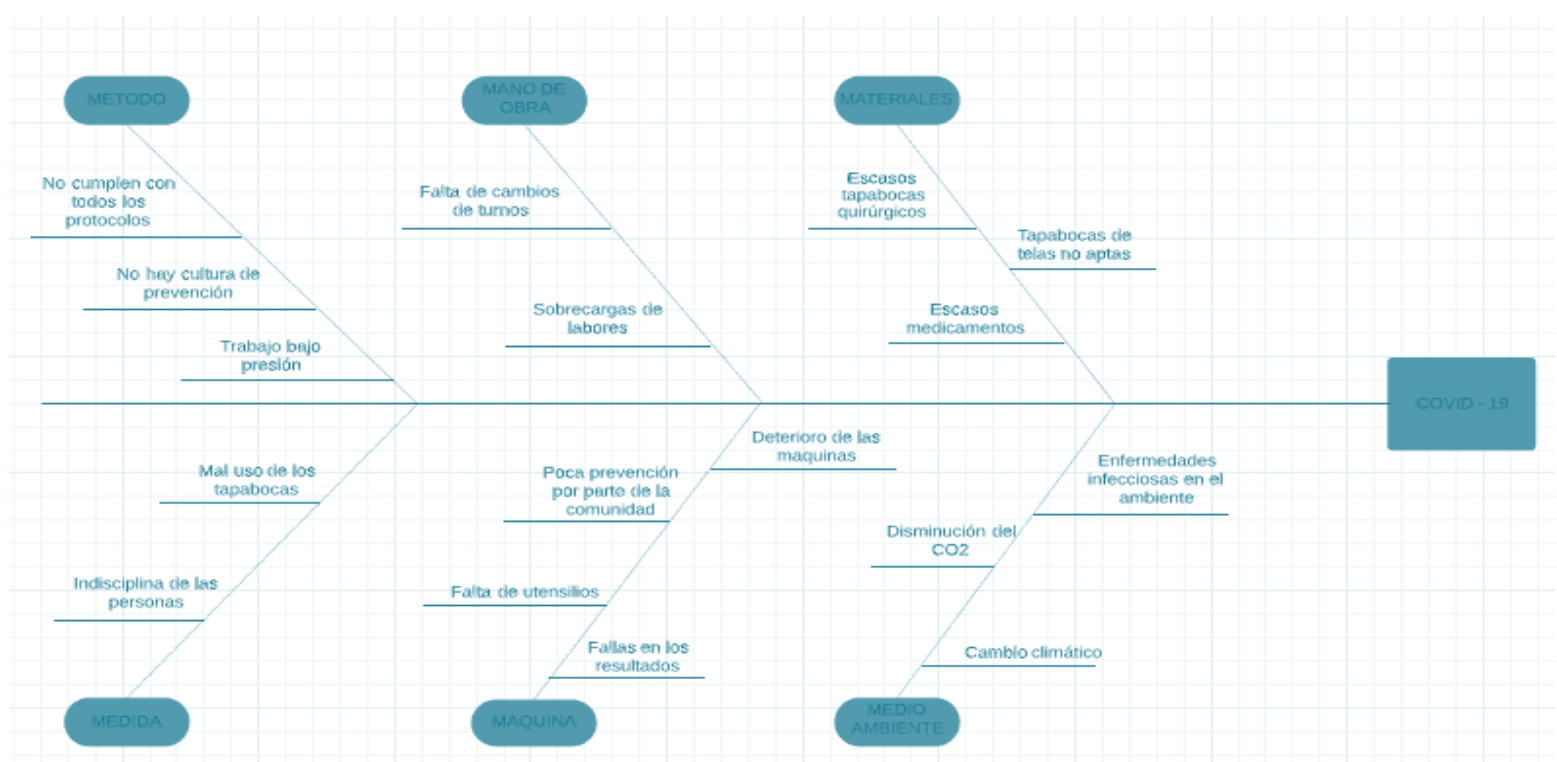

Figura 4. Diagrama de Ishikawa adaptado para el análisis de las causas del crecimiento del coronavirus

Por medio de este diagrama podemos analizar las causas- raíces del COVID-19 y notar aquellos factores que se involucran en la extensión de este virus en la ciudad de Barranquilla. Para ello el gobierno tomo medidas mediante prevenciones de aislamiento, control del lavado de manos, el uso constante y permanente a la hora de salir de los tapabocas y un distanciamiento de dos metros por cada persona. Estas precauciones ayudaron a los ciudadanos a tomar conciencia y a seguir dichos cuidados para bajar el índice de mortandad, debido al gran incremento que se vivió, que fue nefasto para aquellas personas que perdieron algún familiar, amigo o conocido.

(C) The author; licensee Universidad de la Costa - CUC. 


\section{Conclusión}

Por todo lo anterior mencionado se deja en evidencia el impacto que ha tenido este virus en la sociedad, el nivel de casos que tiene, así como la influencia que ha tenido la imprudencia social en el aumento de los casos, las múltiples razones por las cuales esta pandemia se ha vuelto un gran acontecimiento en esta década que dejará marca en la sociedad y en su economía. Esto deja en evidencia la precariedad de nuestra sociedad ante estas enfermedades, las personas no estas preparadas para afrontar una enfermedad de esta magnitud y eso se refleja en las cifras de contagios.

Luego del análisis realizado a través de las herramientas de calidad presentadas anteriormente como fue el uso de las tablas, el histograma, las tablas de tiempo y el diagrama de Ishikawa nos permitió el fácil análisis e interpretación de las estadísticas y número que son claves a la hora de informar y comprender la magnitud de esta catástrofe que se está viviendo en la actualidad

\section{Referencias}

[1] A. Ricaurte Fajardo, V. Hernandez Aguirre y M. Garnica Echeverri, «Características clínicas de las manifestaciones extrapulmonares de la infección COVID-19,» Salutem Sciencia Spiritus, vol. 6, no 1, pp. 116-123, 2020.

[2] J. E. Diaz Pinzon, «Estimación de las tasas de mortalidad y letalidad por COVID-19 en Colombia,» Repertorio de Medicion y Cirugia, vol. 29, pp. 89-93, 2020.

[3] A. Rodriguez Morales y W. E. Villamil Gomez, «Esperando lo inesperado, la pandemia del Milenio...,» Editorial, vol. 13, no 1, pp. 4-7, 2020.

[4] J. Peralta Polo, M. A. Caro Candezano y L. Niebles Núñez, «Dos enfoques matemáticos epidemiológicos para modelar el comportamiento de los decesos causados por el COVID19,» Investigación e innovación en Ingenerías, vol. 8, nº 2, pp. 75-86, 2020.

[5] M. Alcatara Saez, «America Latina y el Covid-19,» Middle Atlantic Review of Latin American Studies, vol. 4, no 1, pp. 16-19, 2020.

[6] C. Bustamante Melendez, D. Amaris Rueda, S. Mora Piña y A. Troncoso Palacio, «Caracterización de contagiados por COVID-19 en el Departamento del Atlántico,» Boletin de Innovacion, Logistica y Operaciones, no 138, pp. 1-35, 2020.

[7] J. Gomez Marin, A. Gonzales y A. Rodriguez Morales, «pandemia covid-19: reflexiones sobre su impacto para la preparación en el control de enfermedades infecciosas en colombia,» Infectio, vol. 24, $\mathrm{n}^{\circ} 3,2020$.

[8] J. J. Garcia Iglesias, J. Gomez Salgado, J. Martin Perera, J. Fagundo Rivera, D. Ayuso Murillo, J. R. Martinez Riera y C. Ruiz Frutos, «Impacto del SARS-CoV-2 (Covid-19) en la salud mental de los profesionales sanitarios: una revisión sistemática,» Revista Española de la Salud Publica, vol. 94, 2020.

[9] P. Rondon Velez, «El tiempo de la Inexperiencia,» Marlas, vol. 4, n 1, pp. 45-48, 2020.

[10] T. Lamprea Barragan, G. Hernandez, C. V. Ospina Cartagena y A. Rivera, «Una medida de los efectos potenciales del Covid-19 en el empleo: el caso de la política de aislamiento preventivo obligatorio en Colombia,» ResearchGate, 2020.

[11] M. Barbosa y D. Montag, «El COVID-19 y su impacto socio cultural:emociones, poderes y nueva solidaridades,» Instituto Universitario de Investigacion en Estudios Latinoamericanos, 2020.

[12] A. Moreno Ariza, J. Hernandez Barrios, I. Alfaro Sarmiento y A. Troncoso Palacio, «Comportamiento Adoptado en América Latina Debido al covid-19,» Boletin de Innovacion, Logistica y Operaciones, vol. 2, $\mathrm{n}^{\mathrm{o}}$ 1, 2020. 
[13] B. N. Morales Contreras y J. J. Palencia Sierra, «Reflexiones del cuidado enfermero en tiempos de Covid-19,» Enfermeria Investiga, vol. 5, nº 3, pp. 71-78, 2020.

[14] A. Urzua, P. Vera Villarroel, A. Caqueo Urizar y R. Polanco Carrasco, «La Psicología en la prevención y manejo del COVID-19. Aportes desde la evidencia inicial,» Scielo, vol. $38, \mathrm{n}^{\circ} 1$, 2020.

[15] A. Campos Mahecha, A. Otaya Tono, M. Garcia, C. Jaramillo Moncayo y C. Wills , «COVID-19: Generalidades, comportamiento epidermico y medidas adoptadas en medio de la pandemia en Colombia,» Acta de Otorrinolaringologia \& Cirugía de Cabeza y Cuello, vol. Abril, pp. 4-13, 2020.

[16] F. G. Manrique Abril, C. A. Agudelo Calderon, V. M. Gonzalez Chorda, O. Gutierrez Lesmes, C. F. Tellez Piñerez y G. Herrera Amaya, «Modelo SIR de la pandemia de Covid-19 en Colombia,» Salud Publica, vol. 22, n 2, Abril 2020.

[17] J. E. Diaz Pinzon, «Analisis de los resultados del contagio del Covid-19 respecto a su distribucion geografica en Colombia,» Repertorio de Medicina y Cirugia, 2020.

[18] D. Alvares Arias, O. Arrieta Cueto, J. Hurtado Rivera, J. Nuñez Mendoza y J. Rico Herrera, «Un breve análisis de la mortalidad del Covid-19 en países de América Latina,» Boletin de Innovacion, Logistica y Operaciones, vol. 2, $\mathrm{n}^{\mathrm{o}}$ 1, 2020.

[19] J. E. Diaz Pinzon, «Estimacion de la prevalencia del Covid-19 en Colombia,» Repertorio de Medicina y Cirugia, vol. 29, pp. 99-102, 2020.

[20] J. Aparicio Barrera, «Análisis espacio-temporal del incumplimiento de normas legales sobre el confinamiento en Colombia por COVID-19,» La Revista Logos Ciencia \& Tecnología desde ahora en el área de conocimiento de las Ciencias Sociales, vol. 12, nº 3, pp. 5-6, 2020. 